Service social

\title{
Le savoir se construit - Une liste de travaux de maîtrise en service social dans trois universités québécoises francophones sur les conditions féminines et masculines
}

\section{Geneviève Martin}

Volume 37, numéro 1-2, 1988

Par-delà les barrières des sexes

URI : https://id.erudit.org/iderudit/706395ar

DOI : https://doi.org/10.7202/706395ar

Aller au sommaire du numéro

Éditeur(s)

École de service social de l'Université Laval

ISSN

1708-1734 (numérique)

Découvrir la revue

Citer ce document

Martin, G. (1988). Le savoir se construit - Une liste de travaux de maîtrise en service social dans trois universités québécoises francophones sur les conditions féminines et masculines. Service social, 37(1-2), 245-252. https://doi.org/10.7202/706395ar
Résumé de l'article

Le savoir se construit d'utilisation que vous pouvez consulter en ligne. 
MARTIN, Geneviève, professeure à l'École de service social de l'Université Laval.

\section{Le savoir se construit - Une liste de travaux de maîtrise en service social dans trois universités québécoises francophones sur les conditions féminines et masculines}

\section{Geneviève Martin}

Depuis quelques années, des étudiantes et étudiants en service social réalisent des travaux de maîtrise se rapportant au thème du présent numéro. La liste ci-dessous se veut un premier effort pour favoriser la consultation de ces travaux et pour rendre hommage à celles et ceux qui ont ainsi allié la démarche académique à leurs préoccupations de transformations sociales.

Les documents ici énumérés, qu'il s'agisse de mémoires, d'essais, d'études thématiques ou de rapports de stage, se retrouvent dans les centres de documentation des trois universités concernées: Laval, Montréal et Sherbrooke. Je tiens à remercier, pour leur précieuse collaboration lors de la constitution de cette bibliographie : Margaret Beatty, professeure au Département de travail social de l'Université de Sherbrooke; Gisèle Legault, professeure à l'École de service social de I'Université de Montréal; et Jocelyn Lindsay, professeur à l'École de service social de l'Université Laval.

\section{I. École de service social, Université Laval}

\section{A. Essais et mémoires de maîtrise (1984-1989)}

AlLARD, Mireille

Maternité et intervention. Expérimentation auprès de femmes enceintes par une approche

S.S., vol. 37, nos 1 et $2,1988$. 
AMPLEMAN, Gisèle et

DUHAIME, Roland

BerNier, Solange

Bilodeau, Dominique et DuMOUCHEL, Suzanne

BoIvin, Julie et MerCIER, Martine

BrousSeAU-GINGRAS, Michèle

Chouinard, Gertrude

Cloutier, Monique

COTtE, Hélène

Drolet, Marie de groupe de service social axée sur la croissance et l'aide mutuelle. Essai de maîtrise, 1986.

Formation à l'intervention en périnatalité en milieux populaires. Une réflexion sur une première session de formation en éducation sanitaire en milieux populaires, dans le domaine de la périnatalité, en mars 1985. Essai de maîtrise, 1986.

Analyse d'une nouvelle pratique sociale et féministe auprès des femmes qui font un retour au marché du travail. Essai de maîtrise, 1984.

L'exploration de possibles dans une articulation entre l'approche féministe et l'approche de conscientisation. Regrouper des femmes anciennement hébergées à la Maison des femmes de Québec. Essai de maîtrise, 1987.

L'une parle, l'autre pas. Analyse discursive du rapport des femmes de milieux populaires à l'idéologie féministe. Mémoire de maîtrise, 1986.

Effets d'un programme d'intervention de groupe avec approche féministe sur l'estime de soi, l'affirmation de soi et l'état dépressif. Mémoire de maîtrise, 1989.

L'utilisation de l'intervention féministe concernant la relation mère-fille chez les adolescentes en besoin de protection. Essai de maîtrise, 1987.

Projet de groupe de développement personnel et professionnel auprès d'intervenantes travaillant avec des adolescentes. Essai de maîtrise, 1988.

Une expérience d'intervention féministe en milieu rural. Essai de maîtrise, 1985.

Intégration de la définition féministe du concept d'autonomie chez les adolescentes usagères du Centre de santé des femmes de la Mauricie. Mémoire de maîtrise, 1987. 
DUBÉ, Julienne

FLEURY, Lorraine

GaGnON, Michel

GaUdreau, Lorraine

GINGRAs-Potvin, Madeleine

GIRARD, Michelle

GOUDREAULT, France

Hébert, Lise

LECLERC, Johanne

LEGAULT, François

LeGault, Suzanne

MoIsan, Marie
Hommes et femmes alcooliques: leurs idées et tentatives de suicide. Mémoire de maîtrise, 1985.

Le café-rencontre du village. Une pratique "féministe conscientisante" avec des femmes de classe populaire en milieu rural. Essai de maîtrise, 1987.

Father-Daughter Incest : A Feminist Analysis of How The Fathers and the Mothers are Described in the Litterature. Mémoire de maîtrise, 1987.

Les attitudes politiques de militants et militantes de classe populaire. Analyse de contenu d'entrevues de groupe. 1985-87. Mémoire de maîtrise, 1987.

Le passage du modèle thérapeutique au modèle de la vie quotidienne en intervention de "petits groupes " auprès des mères célibataires. Essai de maîtrise, 1984.

La réactions des victimes de viol - Une revue de la littérature. Essai de maîtrise, 1985.

Une intervention féministe en groupe ouvert avec une clientèle d'une maison d'hébergement pour femmes victimes de violence. Essai de maîtrise, 1986.

L'utilisation complémentaire de l'intervention féministe et du modèle de réciprocité dans un groupe de femmes aînées. Essai de maîtrise, 1985.

La syndicalisation dans les garderies populaires du Québec de 1969 à 1984. Mémoire de maîtrise, 1986.

Le passage d'un groupe de traitement pour hommes agresseurs à un groupe d'entraide. Essai de maîtrise, 1989.

La relation entre la satisfaction maritale et le niveau d'androgynie chez les jeunes couples du Nord-Est ontarien. Mémoire de maîtrise, 1985.

Pouvoir des femmes face aux hommes dans la famille : une analyse féministe. Essai de maitrise, 1988. 
Olivier, Victoria

RONDEAU, Suzanne

SaINT-HILAIRe, Monique
Les femmes cheffes de famille : contradictions sociales et pistes d'intervention. Réflexion critique suite au stage réalisé auprès des femmes cheffes de famille au Centre de services communautaires de Limoilou. Essai de maîtrise, 1989.

Le maintien et le transfert du changement en travail de groupe. Revue de la littérature et application à une expérience d'intervention féministe auprès d'un groupe de femmes clientes d'une clinique externe de psychiatrie. Essai de maîtrise, 1988.

La composition d'un groupe. Expérimentation auprès d'un groupe de femmes cheffes de famille d'une approche de groupe en service social axé sur la croissance personnelle et l'aide mutuelle. Essai de maîtrise, 1989.

\section{II. École de service social, Université de Montréal}

\section{A. Études thématiques (1983-1986)}

\author{
Alarie, Françoise \\ BourgeoIs, Denise \\ BOUtHILLIER, Monique \\ BrISSETTE, Lyne \\ CORBEIL, Louise \\ De La SABLonnière- \\ Derome, Nicole \\ Demers, Monique \\ GAGNON, Sylvie \\ Girouard, Carmel \\ GuINDON, Nicole
}

La prostitution féminine : une énigme. 1985.

L'inceste père-fille. 1985.

Pauvre à la retraite parce que femme. 1983.

La criminalité des femmes malades mentales. 1983.

Une évaluation de besoins en vue d'implanter un centre d'aide pour les victimes d'agressions à caractère sexuel. 1984.

Les tentatives de suicide chez les femmes adultes. 1985.

Femmes et dépression. 1984.

Les conséquences à long terme de l'inceste père-fille. 1983.

L'homme et le travail. 1984.

Les problèmes de réajustement des femmes suite au divorce ou à la séparation. 1984. 
LAZure, Carole

LeCHASSEUR, Mona

Leroux, Jean-Dominique

LONGPRÉ, Marlène

LORANGER, Sylvia

MAHONEY, Diane

Melanson-Das, Blanche

MONTPETIT, Lucie

Moreau, Micheline

Nadeau, Andrée

PAQUIN, Guylaine

PierRe-Louis, Martine

Soucr, Lyse

TreVINO, Raquel
L'intervention féministe et les femmes violentées: évaluation d'une expérience d'intervention féministe auprès d'un groupe de femmes violentées. 1983.

Le travail domestique et ses interdépendances au travail salarié et à la société marchande. 1985.

La dépression majeure chez la femme adulte Nord-Américaine. 1985.

L'avortement au Québec: lieu de pouvoir absolu de la médecine et des interminables luttes féministes. 1985.

L'abandon scolaire chez l'adolescente. 1983.

Les conjointes brutalisées : pourquoi restentelles? 1985.

La place des organismes populaires dans la lutte contre la violence faite aux femmes. 1983.

Étude du phénomène de la criminalité féminine et de ses causes, des interventions et programmes préventifs pratiques dans ce domaine. 1984.

Carnet de notes sur les garderies ou «maman, je t'aime beaucoup, mais j'aime mieux aller à la garderie ». 1983.

Pour une reconnaissance des femmes au foyer. 1985.

Les femmes violentées qui retournent auprès de leur conjoint. 1985.

Problèmes d'adaptation et d'intégration des femmes immigrantes et répercussions sur la santé mentale. 1985.

Être une femme et une travailleuse : un défi de taille au Québec ou de l'injustice à l'égalité. 1985.

Les problèmes psychosociaux reliés à l'adaptation de la femme immigrante. 1984. 


\section{B. Mémoires de maîtrise (1983-1988)}

Constantin, Rénald

Côté, Marguerite

Fortin, Denise

LORANGER, Sylvia

PARAdIS, Marguerite

SOUCY, Lyse

Turcot, Ginette
Processus de différenciation sexuelle dans l'évolution des thèmes de recherche des étudiants de maîtrise en service social (1942-1982). 1983.

Participation du père aux tâches familiales et développement de l'identité sexuelle du jeune garçon. 1986.

L'entente de garde conjointe suite aux interventions du Service de médiation à la famille de Montréal. 1985.

Implantation et évaluation d'un programme de compétences à des raccrocheuses scolaires du secondaire. 1987.

Les femmes itinérantes : 4 récits de vie. 1987.

Les aspirations des femmes aux postes de cadres dans les services sociaux. 1987.

Le phénomène de la tentative de suicide et les stéréotypes sexuels. 1985.

\section{Rapports de stage (1983-1987)}

BeAuregard, Louise

BONHOMME, Dominique

BOURGEOYs, Denise

Cantin, Marie et

CORBEIL, Louise

DeMERS, Monique

GAGNON, Sylvie

Giroux, Aline

GuAY, Louise et

LeHOUX, Louise
Identité nouvellement définie : passeport de femmes, application d'un modèle d'intervention féministe auprès des femmes de 50-65 ans. 1986.

Se reprendre en main: Groupe de femmes séparées ou divorcées. 1987.

Rapport de stage en intervention féministe. 1985.

Analyse d'un groupe Nous femmes ou une expérience d'intervention féministe. 1983.

Femmes et dépression. 1984.

Rapport de stage en intervention féministe auprès de femmes en difficultés conjugales. 1984.

Intervention féministe et concept d'identité auprès de femmes violentées. 1984.

L'autonomie économique des femmes. 1984. 
GuINDON, Nicole

LABROSSE, Jacinthe

LAMOTHE, Louise

LANCUP, Solange et

SÉGuIN, Jocelyne

LAPIERRE, Lise

LECHASSEUR, Mona

MaHONEY, Diane et

PAQUIN, Guylaine

Moreau, Micheline et Lemieux, Monique

MORIN, Albanie

RiCHARD, Anne et

LAPRISE, Chantal

Sigouin, Danielle
Rapport théorique et pratique de stage en intervention féministe. 1983.

L'intervention féministe : Théorie et application. 1987.

Rapport de stage en intervention féministe : Si on se parlait de notre vécu. 1985.

Analyse de l'expérience du groupe NousFemmes II. 1983.

Regard sur la démission des femmes. 1987.

Intervention féministe auprès de femmes chefs de familles monoparentales. 1984.

Intervention féministe auprès de femmes victimes de violence, secteur nord du CSSMM. 1986.

Intervention féministe auprès de femmes-mères. 1983.

L'autonomie et la solidarité des femmes après leur participation au collectif du divorce. 1983.

L'intervention féministe et les femmes victimes d'inceste dans leur enfance. 1987.

L'intervention féministe auprès de femmes dont le conjoint ou le fils est détenu. 1987.

\section{Département de travail social, Université de Sherbrooke}

\section{A. Essais de maîtrise (1983-1988)}

ASSELIN, France et

Pelletier, Marie

LAFRANCE, Lise
Les facteurs structurels et culturels contribuant à l'exclusion sociale des femmes chefs de famille monoparentale de Pointe-Gatineau. 1984.

L'impact d'un retour au travail pour les femmes. 1987. 
LetTre, Solange et

Pouliot, Hermance

ROYer, Paul
Les femmes dans les coopératives d'habitation et leur participation au mouvement des femmes. 1983.

Étude critique de conscientisation avec les femmes assistées sociales de Sherbrooke, 1982-1985. 1987. 